\title{
The First Ten Years of GRAPE
}

\author{
Daiichiro Sugimoto \\ University of the Air, 2-11 Wakaba, Mihama-ku Chiba 261-8586, Japan
}

\begin{abstract}
GRAPE is a project for a special-purpose computer dedicated to classical many-body problems. Its primary use is for gravitational many-body problem of stellar systems. It was initiated in 1989, and now its performance is in the range of 100 Tera-flops equivalent. As an introduction to this symposium, development of the GRAPE project is reviewed, where motivation to it is the main concern of this paper rather than the computer itself.
\end{abstract}

\section{Introduction}

Gravitational many-body problem deals with dynamical evolution of a system consisting of a large number of elements interacting one another with gravitational force. When the element is regarded as a star, the system will be a (globular) star cluster or a galaxy. When a plural number of the star clusters or of the galaxies are regarded as system consisting of the stars as the elements, the system represents binary globular cluster (Sugimoto \& Makino 1989), colliding galaxies, or group of galaxies. When the element is regarded as a galaxy, the system will describe a cluster of galaxies or the universe. For the sake of simplicity, we shall call the element as the star even if it is a galaxy. The gravitational many-body problem is conveniently called as $N$-body problem where $N$ denotes the number of the elements.

As compared with fluid-dynamic simulation of astronomical objects, the simple $N$-body simulation can handle without any specific recipes even three dimensional structure and/or high density contrast as encountered in colliding galaxies and in a collapsed core of a globular cluster. However, the $N$-body simulation is too much computer intensive, since the number of the (gravitational) forces to calculate amounts to the order of $\mathrm{O}\left(N^{2}\right)$. Though there are some hierarchical algorithms which reduce the necessary amounts of computation to $\mathrm{O}(N \ln N)$, the simple $\mathrm{O}\left(N^{2}\right)$ algorithm is robust in any situations of very high density contrast, in particular. This is the reason why we need a computer much faster than commercially available one at practically reasonable cost.

\section{Short History}

To meet this requirement, we proposed in 1989 the concept of GRAPE as shown in Figure 1 (Sugimoto et al. 1990). It consists of a programmable host computer and a GRAPE. As shown in Figure 1 the host sends to the GRAPE positions of 
all the stars, $\boldsymbol{x}_{i} ; i=1,2, \ldots, N$. The GRAPE calculates the force per mass $\boldsymbol{F}_{k}$ exerted onto the $k$-th star from all the other stars, $i=1,2, \ldots N$, and returns it to the host. They are expressed as follows:

$$
\begin{aligned}
\frac{\mathrm{d}^{2} \boldsymbol{x}_{k}}{\mathrm{~d} t^{2}} & =\boldsymbol{F}_{k}, \\
\boldsymbol{F}_{k} & =\sum_{i} \boldsymbol{f}_{k i}, \\
\boldsymbol{f}_{k i} & =G m_{i} \frac{\boldsymbol{r}_{k i}}{\left(\boldsymbol{r}_{k i}^{2}+\varepsilon^{2}\right)^{3 / 2}}, \\
\boldsymbol{r}_{k i} & =\boldsymbol{x}_{i}-\boldsymbol{x}_{k},
\end{aligned}
$$

where $m_{i}$ is the mass of the $i$-th star, $G$ is the constant of gravity, and $\varepsilon$ is a small smoothing factor to avoid divergence.

To integrate equation (1) by one time step, the order of $\mathrm{O}\left(N^{2}\right)$ operations are necessary on the GRAPE, while $\mathrm{O}(N)$ operations are performed on the host and the same order of communication is required between the host and the GRPAE. Therefore, the calculation speed of the host and the communication bandwidth may be slower than the GRAPE by a factor of $\mathrm{O}(N)$. In other words, it is required and it is enough to make the GRAPE faster than the host and the communication by a factor of $\mathrm{O}(N)$.

Such a high speed of the GRAPE was realized by constructing a pipeline processor to calculate the gravitational force $\boldsymbol{f}_{k i}$ for $i=1,2, \ldots, N$ with a given value of $k$ by feeding $\boldsymbol{x}_{i}$ consecutively to the pipeline, and to sum them up to obtain $\boldsymbol{F}_{k}$. Thus we named it as GRAvitational PipelinE. To speed up the computation a method of table look-up and interpolation were extensively used where, of course, the accuracy of the resultant values was extensively investigated to secure.

Early GRAPEs are described in Ebisuzaki et al. (1993). Here and hereafter, only the most fundamental paper is cited. See also papers referred therein or in more recent papers of related topics that will be cited afterwards. We constructed a prototype machine GRAPE- 1 in 1989 by wiring LSI chips sold in market. Its cost was only about two thousand US dollars. Its performance was as high as 240 Mega-flops equivalent that was as fast as a supercomputer of those days. Here, the speed was denoted with the word of equivalent since the number is expressed only with 16-bit fixed-point format. (The bit-length of the variables was different among variables to optimize the final result of the

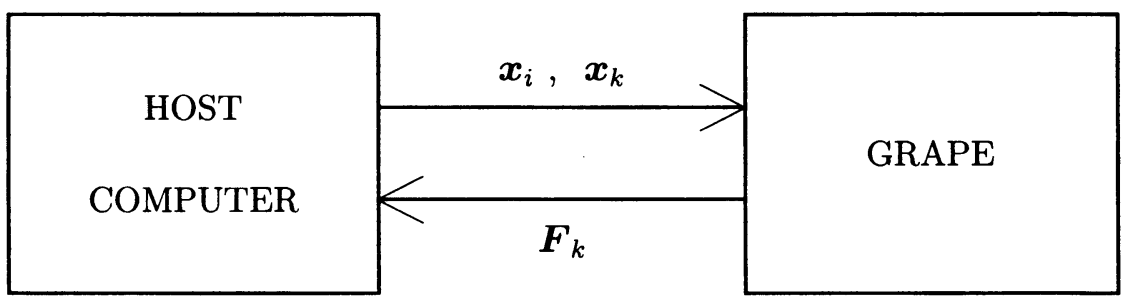

Figure 1. Basic concept of GRAPE system 
force; the summation of equation (2), for instance, was performed with 48-bit fixed-point format.) It was on a single board.

In 1990 we put all the logic, that had been contained on the board of GRAPE-1, into one customized LSI chip, and constructed GRAPE-3 where 48 of such LSIs were connected in parallel on two boards of size $40 \mathrm{~cm} \times 37 \mathrm{~cm}$ each. Its speed was 14 Giga-flops equivalent with 20 bit and 56 bit fixed-point formats. It was extensively used in simulating colliding galaxies etc. not only in our laboratory but also in other domestic and foreign institutes as was discussed by Piet Hut in the preceding paper of this symposium.

The GRAPE series of odd serial number are the machine of low accuracy models having a shorter word length than 32 bits. Nevertheless, it is enough for simulating stellar dynamics of collisionless system. Its present version is GRAPE-5. Its one unit (one board with power supply) loads 8 newly customized LSIs (ASIC - Application Specific Integrated Circuits), each of which has 2 real pipelines used in the mode of 12 virtual pipelines. Its one unit attains the performance of 38.4 Giga-flops, which corresponds to 1.28 Giga force calculations per second. Sixteen units of them are now working at the National Astronomical Observatory of Japan in parallel connection attaining at the maximum speed of 600 Giga-flops equivalent.

In order to simulate dynamical evolution of collisional system such as core collapse of a globular cluster, however, we need higher accuracy of 32 and 64 bit format. GRAPE series of even serial numbers are machines of such accuracy. GRAPE-2 was just a prototype machine of wired ICs. In 1995 we completed GRAPE-4 that attained at the peak speed of 1.08 Tera-flops. Its details are described in Makino et al. (1997).

Each pipeline of GRAPE-4 calculates not only the force itself but also the time derivative of the force by using coordinates and velocities of the stars. One pipeline is put in one HARP (Hermite AcceleRator Pipeline) LSI chip. Eight HARP chips are mounted on an MCM (Multi-Chip Module), and six MCMs are mounted on a processor board (PB). Nine PBs are connected to form one cluster in a box of size $57 \mathrm{~cm}(\mathrm{~W}) \times 65 \mathrm{~cm}(\mathrm{D}) \times 109 \mathrm{~cm}(\mathrm{H})$ including the power supply. Four clusters form the GRAPE-4 system, which has 1728 (= $8 \times 6 \times 9 \times 4)$ pipelines in parallel. In actual, $47 / 48$ of the pipelines remained active which had avoided any defects from manufacturing process.

Sustained speed of the GRAPE-4 system is of the same order of magnitude as its peak speed though the former depends on problems to compute, i.e., on the workload of communications between the host computer and the GRAPE-4. In the GRAPE-4 a specially designed ASIC is also implemented on each PB that treats algorithm of multiple time steps (Aarseth 1985). Thus, it can calculate even a system of extremely high density contrast including binary stars formed therein. The project for the GRAPR-4 was conducted with the funding of 2 $\times 10^{8}$ Japanese Yen (1.6 Million US dollars on those days) from the Grant-in Aid for Specially Promoted Research (04102002) of the Japanese Ministry of Education, Science, Sports and Culture.

In the spring of 1997, I retired from the University of Tokyo because of its retirement age. On those days we discussed possible future for the GRAPE project. We considered two directions for its extension; one aiming at the speed of Peta-flops but limited to astrophysical applications with gravitational inter- 
actions, and the other aiming at applications for wider subjects, in particular, molecular dynamics of protein and others. Junichiro Makino succeeded the former under the grant of the Research for the Future Program (RFTP) of the Japan Society for Promotion of Science (JSPS), and Toshikazu Ebisuzaki succeeded the latter at Physical and Chemical Research Institute (RIKEN). Papers will be presented in this Symposium from these projects.

\section{Physics of Self-Gravitating Systems}

\subsection{Evolution of a Star}

A star is a typical self-gravitating system. It evolves by changing its internal structure. After its birth from interstellar gas cloud, the star makes a gravitational contraction and settles itself on the main sequence with hydrogen burning (depending on its mass). After hydrogen is exhausted in the central core of the star, its core contracts gravitationally while its envelope expands greatly. Thus the star evolves to a red giant. Thereafter, the core experiences the nuclear burning phase and the gravitational contraction phase, repeatedly. According to the rise of the central temperature, different nuclear burning phases halt the gravitational contraction of the core. They are, successively, helium-burning phase, carbon-, oxygen, neon-, magnesium-, and silicon burning phases which finally lead to formation of an iron core and then supernova explosion (depending on the initial mass of the star).

This process of stellar evolution can be regarded as formation of structure or emergence of diversity, where spatial differences in temperature and density through the interior of the star are greatly enhanced. In particular, the density difference between the core and the envelope of a red giant star grows even to the factor of more than $10^{12}$. This phenomenon can be regarded as segregation of the system into a high density, low specific entropy core and a low density, high specific entropy envelope, i.e., into the core-halo structure.

Such segregation or, in other words, deviation from uniformity or emergence of diversity would look against the second law of thermodynamics. However, the system is different from a standard one in thermodynamics, i.e., from noninteracting gas in a box. The star is an open system that sheds off its entropy with energy to outside of the system, i.e., to the interstellar space. It is in non-equilibrium state in the sense that temperature and density gradients are maintained with associated heat flow (Sugimoto et al. 1981). In short, it is a nonequilibrium, open system in (quasi-) steady state, which is maintained through the negentropy input according to a wording by Schrödinger. It is realized as the difference of the low (specific) entropy input with energy corresponding to the higher temperature (the nuclear burning or the gravitational energy release) from the high entropy output with the same amount of energy corresponding to the lower temperature (stellar light output).

\subsection{Gravothermal Catastrophe}

Idealized model of the stellar gravitational contraction is the isothermal gas sphere with which Antonov (1962) and Lynden-Bell and Wood (1968) discussed gravothermal catastrophe. It is ideal in the sense that the system of the gas 
sphere is enclosed with an adiabatic spherical wall of radius $R$, and we can discuss the effect of gravitation separately from the openness of the system differently from the star itself.

In such a model the gas sphere is in hydrostatic equilibrium in the sense that there is no mass motion of the gas, and it is in thermal equilibrium in the sense that there is no heat flow in the isothermal gas sphere. If we solve differential equations for hydrostatic equilibrium with a value of the isothermal temperature assumed, we obtain density distribution as a function of radial distance $r$ from the center $(r=0)$ to just inside the wall $(r=R)$. Of particular importance is the density contrast which is the ratio of the density at the center $\rho_{\mathrm{c}}$ and the density just inside the boundary wall $\rho_{\mathrm{b}}$, i.e.,

$$
D=\frac{\rho_{\mathrm{c}}}{\rho_{\mathrm{b}}} .
$$

There is a critical value of the density contrast,

$$
D_{\text {cr }}=709 \text {. }
$$

When $D<D_{\mathrm{cr}}$, the equilibrium solution is at the entropy maximum as expected from the limit $D \rightarrow 1$ to the standard thermodynamics. We shall call such solution as thermal state. When $D>D_{\mathrm{cr}}$ on the other hand, effect of selfgravity is dominant and the equilibrium solution is at the entropy minimum. We shall call it as gravothermal state.

Stability of such states is more clearly understood by means of linearized stability theory (Hachisu \& Sugimoto 1978). We apply perturbation $\delta \sigma(\phi)$ to the distribution of specific entropy $\sigma(\phi)$, where $\phi$ is the mass fraction of the gas contained within the sphere of radius $r$. Then, we can solve an eigen-value problem to obtain growth and/or damping of eigen modes. Its fundamental mode, which has one node on $\delta \sigma(\phi)$, is the most dangerous one against stability. It is unstable for the equilibrium solution of $D>D_{\mathrm{cr}}$.

This instability proceeds as follows. When the specific entropy (heat) is removed, say, from a region 1 at $\phi_{1}$ and deposited onto another region 2 at $\phi_{2}$, the temperature at the region 1 drops by $\delta T_{1}^{(\mathrm{rem})}<0$ and the pressure thereof drops. When $\phi_{1}<\phi_{2}$, the pressure drop at $\phi_{1}$ leads the gravitational contraction and the resultant (adiabatic) compression makes the temperature thereof rise by $\delta T_{1}^{(\text {comp })}>0$. When the latter effect overcomes the former, the total change in the temperature $\delta T_{1}=\delta T_{1}^{(\mathrm{rem})}+\delta T_{1}^{(\mathrm{comp})}$ becomes positive. For the region 2 , vice versa. We call such situation that the gravothermal (effective) specific heat is negative. In such situations a temperature gradient spontaneously appears and grows to help more heat transferred to the same outward direction, which is associated with further gravitational contraction of the inner region.

If the heat is initially removed from the outer region 2 and deposited onto the inner region 1 , the discussion above are reversed, and heat continues to flow inward direction, which is associated with expansion of the inner region.

The discussion above deals with the linearized stability, where the both directions of the heat flow and the contraction/expansion are equivalent. However, they are different in non-linear regime. When the initial heat flow was inwardly 
directed, the core expands and the density contrast decreases. When it becomes lower than $D_{\mathrm{cr}}$, stability is resumed. Then the system evolves to resume another isothermal state corresponding to a local maximum of entropy of the system, though its isothermal temperature is lower and the entropy of the system is higher than those at the initial isothermal state. The system has transited from a gravothermal state with a local minimum of entropy to a thermal state with a local maximum of (and yet higher) entropy (Hachisu et al. 1978).

When the initial heat flow was directed outward, on the other hand, the core contracts and the density contrast increases. The system becomes more and more gravothermally unstable. Lynden-Bell and Wood (1968) named this gravothermal instability of finite amplitude as gravothermal catastrophe. This naming is appropriate, because there is no state of entropy maximum (extremum) in this side of the system. The entropy of the system increases indefinitely, which is associated with contraction of the core to infinite density in a finite time. This holds within the Newtonian regime of gravity. If we take account of the general relativity, it ends up at the entropy of a black hole.

\subsection{Dynamical Evolution of Stellar Systems}

In the context of stellar evolution, the gravothermal catastrophe is the same process as the gravitational contraction of the star. The system is open in the case of the star and the star is always at a stage of finite amplitude of the gravothermal catastrophe.

In the case of the gas sphere discussed in the preceding subsection, the system is enclosed by the adiabatic wall and closed to the outer space. The volume inside the wall stays constant. Therefore, the contraction of the inner region of the system has to be associated with the lowering of the density in the relatively outer region. Thus the system segregates into two subsystems, i.e., the dense, low entropy core, and the less dense, high entropy outer region (halo). When seen from the core, it is open to the outer space (halo). The core behaves similarly to the gravitationally contracting star that is open to the outer space.

This gravothermal catastrophe was considered also as a model of core collapse of a globular cluster (GC for short in what follows). In this relation, the following questions had been asked. The GC does not consist of the gas. Instead, it consists of discrete stars, and yet the stars are rather collision-free in the halo region though they are gravitationally bound and confined in a finite volume. What is the difference between the gaseous, collision dominant gravothermal model and the collision-free GC? To answer this question, Fokker-Planck-type equations were solved for dynamical evolution of the GC. The answer was as follows. There is no essential difference so far as the most essential point is concerned, i.e., the system is gravothermally unstable and the gravothermal catastrophe proceeds to the infinite central density. Here, it should be remarked; the Fokker-Planck-type model describes the discreteness in collisions (momentum transfer) but the medium is continuous corresponding to the infinite number of the star.

Of course, there are some differences between the stellar gravitational contraction and the gravothermal catastrophe of GC. One of the largest differences is the mass of the resultant collapsed core. In the stellar evolution, the mass of the core and the resultant black hole is comparable to the mass of the star. On 
the other hand, the mass of the collapsed core of the GC becomes smaller and smaller as the core collapse proceeds.

This difference comes from the different mode of energy transport, which promotes progress of the gravothermal catastrophe. In the case of the stellar interior, energy is transported by photons. Therefore, the time scale of energy transport is shorter in the halo (envelope region) than in the core. This implies that information of the core well reaches the halo any time, i.e., the halo knows thermal situation of the core and the lower part of the halo can contract together with the core.

In the case of the GC, on the other hand, heat is transported through collision of the particles (discrete stars), and its time scale is shorter in the core than in the halo. Thus the halo does not know thermal situation of the core any more, and the core contracts practically independently from the envelope. Moreover, the time scale in the core becomes shorter and shorter as the core contracts. Thus the contracting part of the core becomes smaller and smaller in mass. This mechanism explains the smallness of the mass fraction of the collapsed core in the GC.

In this relation, it is interesting to note the followings. In the gravothermal collapse of the GC, the temperature distribution in the shrinking core can be regarded almost isothermal (though there almost remains the initial temperature gradient in the halo). This is the reason why the concept and the criterion for the stability in the linearized regime, i.e., for the isothermal gas sphere, continue to hold as a good approximation when it is applied to the shrinked core. Therein, the core is a system enclosed by the bottom of the halo, which corresponds practically to adiabatic wall when seen with the time scale of the core.

\subsection{Comparison with Theory of Stellar Structure and Evolution}

The discussion in the preceding subsection indicates that the most essential part of evolution of the stellar system may be understood by consulting with the theory of stellar structure and evolution as a well-known theory for selfgravitating systems. It tells that the essential point for the gravothermally unstable nature of the self-gravitating system is ascribed to 1) equation of state of ideal gas type, and 2) spherical configuration.

As for the equation of state, counter examples are electron degenerate gas or neutron gas; white dwarf and neutron star do not show the negative (effective) specific heat of the system and are gravothermally stable (thermal system). Here, it is noticed that the case of stellar dynamics corresponds to the case of the ideal gas since the rate of momentum transfer and the energy of random motion of the constituent stars may be regarded as pressure and temperature, respectively.

With the equation of state of ideal gas type, the gravothermal specific heat becomes negative for the spherical configuration as seen from Virial theorem for stellar structure. More refined analysis was obtained by normal mode analysis for the gravothermal instability (Hachisu \& Sugimoto 1978).

Role and Implication of the spherical configuration are typically seen in evolution of the star. When the star evolves to a red giant (Sugimoto \& Fujimoto 2000), the stellar core contracts but the radius of the core must not contract any more. At the outer edge of the stellar core, there is a hydrogen-burning 
shell and its temperature should not exceed the hydrogen-burning temperature. When the hydrogen burning is active, the radius of the burning shell should stay almost constant to avoid higher temperature. It plays a role similar to the wall of constant radius in the model of the gravothermal catastrophe though it is an isothermal wall (heat reservoir at a constant temperature) instead of the adiabatic wall.

Therefore the contraction of the inner part of the core is associated with the lowering of the density just inside the hydrogen-burning shell. It implies the lowering of the pressure thereof. With this lowered pressure, the same mass of the envelope should be sustained against the gravity.

Two different types of possible solutions should be examined for this problem. The first is that the edge of the core contracts to raise the pressure thereof. This type of solution, however, is not realized since the temperature at the hydrogen-burning shell can not be raised appreciably. The second is that the bulk mass of the envelope is pushed outward where the gravity is weaker so that the envelope can be sustained even with the lowered pressure. This second type of solution can be realized for the spherical configuration since the gravitational force depends on the radial distance $r$ from the stellar center as $r^{-2}$. The hydrogen shell burning supplies energy, which is required to push the envelope away, by raising its temperature only slightly (strong temperature dependence of the nuclear energy generation rate) only for a short time.

Another interesting process is thermal pulse of nuclear shell burning. During stellar evolution, thin helium zone is formed surrounding a carbon-oxygen core, for instance. It is thin because it extends only $10^{-2}$ in mass fraction, for instance. Initially, it extends spatially only by $(\Delta r / r) \sim 10^{-2}$, for instance, and it is spatially thin also. Because of small $\Delta r / r$, its configuration is rather plane-parallel than spherical, and, therefore, the effective specific heat thereof is positive even under the gravitational field (practically constant gravitational acceleration). When the helium burning begins in such a thin shell, it makes runaway since the heat released from the nuclear burning simply raises the temperature of the shell (no gravothermal effect) and the nuclear burning rate is increased.

When this runaway (instability) of the nuclear shell burning has grown to a certain finite amplitude, the helium zone expands appreciably since the heat released from the nuclear energy generation piles there up. (Even when the convection has developed, the convective zone can not invade appreciably into the outer shells in the time scale of the energy input of the runaway; the initial specific entropy gradient was too steep in mass-wise and it inhibits the isentropic convection from invading to the outer shells in such a short timescale.) The energy piled up makes the helium zone expand greatly.

Then, the value of $\Delta r / r$ becomes appreciably large and the configuration becomes rather spherical than plane-parallel. This turns the effective specific heat of the burning shell to be negative, i.e., to the gravothermal one. Afterwards, the temperature drops despite further nuclear energy input. Since the time scale becomes longer and longer, heat is dissipated from the extended helium zone. It leads gradual contraction of the helium zone. The temperature of the helium zone becomes low enough and the helium burning becomes extinguished. Finally the helium zone resumes to its initial thin, plane-parallel 
configuration. This makes one cycle of non-linear oscillation (Sugimoto \& Fujimoto 1978). Repetition of this cycle is described approximately as a limit cycle. Here, we have seen typically the difference of implications between the plane-parallel and the spherical configurations.

\subsection{Gravogyro Catastrophe}

In this subsection, we shall consider rotating systems such as spiral galaxies. For gravitationally bound systems a correspondence holds between rotational and thermal properties. For the gravothermal catastrophe the important quantities are the temperature $T$ and the specific entropy $s$. Change in thermal energy per unit mass is described by $T \mathrm{~d} s$. Correspondingly for the rotating system, the change in the rotational energy per mass at a fixed point $r$ is $\omega \mathrm{d} j$, where $\omega$ is the angular velocity and $j$ is the specific angular momentum. Here we notice that the intensive quantities $T$ and $\omega$ correspond each other, and the extensive quantities $s$ and $j$ do.

In the gravothermal instability, the temperature gradient spontaneously appears on an equilibrium isothermal solution. Correspondingly, a differential rotation, i.e., a gradient of the angular velocity, spontaneously appears under certain conditions on the contribution of the self-gravity as compared with the rotational energy and thermal energy. This instability begins even for a system which is gravothermally stable. Hachisu (1979) found such instability and named as gravogyro catastrophe.

It grows quite similarly to the gravothermal catastrophe. Once the gradient in $\omega$ ( $T$ in the case of the gravothermal catastrophe) appears, it helps a flow of $j(s)$ from high to low $\omega(T)$ regions. It proceeds as follows. The region, where the specific angular momentum (specific entropy) is deposited, gets higher $\omega(T)$ in the first place. Then, the centrifugal force (pressure) becomes higher and the region expands with keeping $j(s)$ constant, which results in a decrease of $\omega(T)$. If the effect of the latter change overcomes the former, the total change in $\omega(T)$ becomes negative. Such situations are described in terms of negative gravogyro moment of inertia (negative gravothermal specific heat), which promotes the instability.

The gravogyro instability may be related for a rotating system to be segregated into two subsystems, one in the central region with low specific angular momentum and the other in the outer region with high specific angular momentum. Examples may be the segregation into the bulge and the disk in a spiral galaxy, and the proto-sun and proto-solar nebula in a proto-planetary system.

Further study of such systems to their non-linear regime is numerically complicated, mainly because the system has three-dimensional structure. In addition, the gravogyro nature is coupled with gravothermal situation (Hachisu 1982). Moreover, as discussed in the preceding subsection, the gravothermal instability is weakened in a system that is somewhat flattened by rotation, though it is not described yet quantitatively.

\section{Post-Collapse Evolution of Globular Cluster}

Theory and numerical computation of the gravothermal catastrophe indicate that the central density becomes infinite in a finite time. This finite time is 
called as the core collapse time and estimated as about 15 times the half-mass relaxation time. Nevertheless, observation shows that there are many GCs of age older than their core collapse time.

This implies that GC survives even beyond the core collapse, and such phase was named as post-collapse evolution. In the collapsed core the stellar density is high and stellar collisions take place to form binary stars. When another star collides the binary, super-elastic collisions are more probable than inelastic (They yield also the segregation in energy space). Through the super-elastic collision, the binary becomes more gravitationally bound and the colliding star gets the corresponding energy (For the case of exchange collision the escaping star has higher energy than the incident star). This makes heating of the field stars and thus of the mean field of the GC. This process is interesting also in the sense of segregations, i.e., to the gravitationally bound binaries and relatively high-energy field stars. In other words, the two-body correlations grow in the system with the total energy conserved.

For the dynamical structure of GC this works as energy input and thus this process is often called as binary heating. This looks similar to nuclear energy generation in a star that halts the gravitational contraction. In the case of the star, the nuclear energy input makes the core expand and the central temperature is lowered because of the negative gravothermal specific heat of the core. Then the nuclear energy generation rate is slowed down greatly because of its strong dependence of the nuclear reaction rate. This mechanism well controls the nuclear energy generation rate, and the star enters a stable nuclear burning phase.

In the case of GC the situation is different despite the same negative gravothermal specific heat. The binary heating rate is only weakly dependent on the density and on the temperature as compared with the dependence of the nuclear energy generation rate on the temperature.

Another important difference is that the temperature gradient in the collapsed core is so weak that it is almost isothermal as noted in subsection 3.3. Therefore, a small expansion of the central region due to the binary heating coupled with the negative gravothermal specific heat easily reverses the temperature gradient, i.e., the temperature becomes decreasing to the inward direction. This triggers the inward heat flow, i.e., the gravothermal expansion. Because the core is gravothermally unstable, this inward heat flow and the expansion grow unstably to finite amplitude even when the binary heating becomes negligible any more due to the lowered stellar density in the core. Here, it should be noted that the binary heating does not drive the expansion but has only triggered it.

When the density of the core drops down to a certain value, the time scale of the gravothermal expansion becomes relatively long so that the heat transport near the core edge to the halo can not be regarded as negligible any more. This stops the growth of the gravothermal expansion, and afterwards the heat outflow from the core to the halo leads the core back to the gravothermal contraction.

Such expansion and contraction make non-linear oscillation. We calculated evolution of GC in such situations with a gaseous model by using an approximate binary heating rate (Sugimoto \& Bettwieser 1983). We found a non-linear oscillation and named it as gravothermal oscillation. It showed the form of recurrent pulses, though somewhat with irregular periods, similar to the recurrent flashes 
of helium shell burning in a star. We thought the occurrence of such oscillation was well understandable to be reasonable from the theory of stellar structure, i.e., of physics of typical self-gravitating systems (Bettwieser \& Sugimoto 1984).

This understanding by one of the stellar evolutionists (Sugimoto 1986) suffered from strong challenges from stellar dynamicists. Their points were as follows.

1. GC is a discrete system consisting of finite number of stars.

2. Moreover, stellar collision is not so frequent as atomic collision in stellar interior.

3. Therefore, the star that has got kinetic energy through the super-elastic collision easily escapes from the GC before depositing its energy to the mean field of the GC.

4. In the field of stellar dynamics the equivalence of the gravothermal expansion and the gravothermal contraction in linear regime was not well-paid attention to.

These points could wash out the occurrence of the oscillation. Thus, I confronted with the burden to show, which is more important, the self-gravitating nature or the discreteness of the system.

Some works were done afterwards by means of the gaseous models with better approximations to the binary heating rate, and of the Fokker-Planck treatment of stellar dynamics. However, $N$-body simulations were needed to clarify the situation with appreciably large number $N$ of the stars. This was one of the strongest motivations to me for constructing ultra-fast computers. If I am allowed to refer only to the result, $N=32000$ body simulation with GRAPE4 clearly showed, avoiding statistical noises, the existence of the gravothermal oscillation (Makino 1996). Here, the scaling of physical results from $N=3000$ through 32000 was also shown to be consistent with prediction form the theory. Of course, the discreteness of the system modifies the oscillation, but essential physics of the oscillation was understood in terms of the self-gravitating nature of the system as described above in the preceding section.

\section{Ultrafast $\mathbf{N}$-body Computers for Physical Understanding}

Discussion in the preceding sections shows the following motivations for constructing a further ultrafast N-body machine to solve:

- effect of discreteness in stellar dynamical systems,

- three-dimensional structure and evolution of rotating systems, merging binary GCs, collision and merging of galaxies, etc.,

- evolution of the system with multiple time scales, e.g., effect of binary stars in a stellar system, and evolution of cluster of galaxies for which stars are collisionless in a galaxy but galaxies are collisional in the cluster. 
When an ultrafast computer is available, one is apt to be tempted into constructing more detailed model than exploring fundamental physical processes involved. However, it seems, at least for me, more important to explore fundamental physical processes such as competition between the discreteness and the self-gravitating nature, effect of rotation in terms of gravogyro catastrophe, separability or non-separability of different time scales, separability or interaction between non-coherent collisions between the stars in a galaxy and coherent scattering of galaxies as larger entities, etc. The gravitational many body problems are, I think, a typical problem of long-range interaction (effective range of the gravitational force is infinity) of coupling without shielding differently from the electromagnetic Coulomb interaction, and yet of simple functional form of the interaction which allows quantitative treatment (though numerically). It will not be too ambitious to say that it will promote not only explaining of astrophysical phenomena but also understanding of non-linear phenomena of structure formation in more generalized terms, which will be one of the main problems of science in the 21-th century.

\section{References}

Aarseth, S. J. 1985, in Multiple Time Scales, ed. J. U. Brackhill \& B. I. Cohen (New York: Academic Press), 377

Antonov, V. A. 1962, Vest. Leningr. Gos. Univ., 7, 135

Bettwieser, E. \& and Sugimoto D. 1984, MNRAS, 208, 493

Ebisuzaki, T., Makino, J., Fukushige, T., Taiji, M., Sugimoto, D., Ito, T., \& Okumura, S.-K. 1993, PASJ, 45, 269

Hachisu, I. 1979, PASJ, 31, 523

Hachisu, I. 1982, PASJ, 34, 313

Hachisu, I. \& Sugimoto, D. 1978, Progr. Theoret. Phys. (Kyoto), 60, 123

Hachisu, I., Nakada, Y., Nomoto, K. \& Sugimoto, D. 1978, Progr. Theoret. Phys. (Kyoto), 1978, 393

Lynden-Bell, D. \& Wood, R. 1968, MNRAS, 138, 495

Makino, J. 1996, ApJ, 796

Makino, J., Taiji, M., Ebisuzaki, T., \& Sugimoto, D. 1997, ApJ, 480, 432

Sugimoto, D. 1986, in IAU Symp. 113, Dynamics of Star Clusters, eds. J. Goodman and P. Hut (Dordrecht: Reidel), 207

Sugimoto, D. \& Bettwieser, E. 1983, MNRAS, 204,19P

Sugimoto, D. \& Fujimoto, M. Y. 1978, PASJ, 30, 467

Sugimoto, D. \& Fujimoto, M. Y. 2000, ApJ, 538, 837

Sugimoto, D. \& Makino, J. 1989, PASJ, 41, 1117

Sugimoto, D., Eriguchi, Y., \& Hachisu, I. 1981, Progr. Theoret. Phys. (Kyoto), Suppl. 70, 154

Sugimoto, D., Chikada, Y., Makino, J., Ito, T., Ebisuzaki, T., \& Umemura, M. 1990, Nature, 345, 33 